\title{
ON EXTENDING THE LEBESGUE INTEGRAL
}

\author{
JAMES FORAN
}

\begin{abstract}
In this paper continuous extensions of the Lebesgue Integral which integrate their almost everywhere approximate derivatives are considered. First, those extensions which are generated by a single function are characterized. Then the largest extension which is contained in all maximal extensions is considered and shown to contain the wide sense Denjoy Integral. Finally, properties are given which characterize this largest extension.
\end{abstract}

The purpose of this paper is to consider properties of extensions of the Lebesgue Integral. Such extensions will be considered to be a collection of primitives that are continuous and integrate their almost everywhere approximate derivatives. The extensions will be assumed to be finitely additive and closed under linear changes of variable. Thus the Lebesgue Integral is the collection of absolutely continuous (AC) functions. Given a function $F$ and a point $x_{0}$, if there is a set $E$ such that $E$ has $x_{0}$ as a point of density and $\lim _{x \rightarrow x_{0} x \in E}\left(F(x)-F\left(x_{0}\right)\right) /(x-x)$ exists, then the value of this limit defines the approximate derivative of $F$ at $x_{0}$ and is denoted by $F^{\prime}$ ap $\left(x_{0}\right)$. A collection of continuous functions defined on the real line (although frequently restricted to an interval $I$ ) will be considered to be an integral $\mathbb{Q}$ provided

(1) $F \in \mathbb{Q}$ implies $F^{\prime}$ ap exists a.e.,

(2) $F \in \mathbb{Q}$ and $F^{\prime}$ ap $=0$ a.e. implies $F \equiv C$,

(3) $F \in \mathbb{Q}, I=[a, b]$,

$$
G(x)= \begin{cases}F(a) & \text { if } x<a \\ F(x) & \text { if } a<x<b \\ F(b) & \text { if } b<x\end{cases}
$$

implies $G \in \mathbb{Q}$,

(4) $F \in \mathbb{Q}, a, b \in \mathbf{R}$ implies $F(a x+b) \in \mathbb{Q}$,

(5) $F \in \mathbb{Q}, G \in \mathbb{Q}, c \in \mathbf{R}$ implies $F+G \in \mathbb{Q}$ and $c F \in \mathbb{Q}$.

The integral should be thought of as giving rise to the formula $\int_{a}^{b} F^{\prime}$ ap $(x) d x=$ $F(b)-F(a)$. The uniqueness of the integral follows from (2), existence on subintervals from (3), the ability to form linear change of variables from (4), and the finite additivity of the integral from (5).

Since only those integrals that include the Lebesgue Integral are under consideration, it is convenient to adopt the following notation: $\langle F, A C\rangle$ will denote the set

Received by the editors November 27, 1979.

1980 Mathematics Subject Classification. Primary 26A42, 26A24, 28A15, 26A39.

Key words and phrases. Lebesgue Integral. 
of all functions of the form $\sum_{i=1}^{n} c_{i} \cdot F\left(L_{i}\right)+G$ where $c_{i} \in \mathbf{R}, L_{i}$ are linear changes of variable, and $G$ is absolutely continuous. Thus $\langle F, A C\rangle$ is the smallest vector space containing $F$ and all linear changes of variable of $F$ and the AC functions.

If $F$ is any continuous function that is approximately derivable almost everywhere and each $G \in\langle F, A C\rangle$ satisfies Lusin's condition (N) (i.e., the images under $F$ of sets of measure 0 are all of measure 0 ), then $\langle F, A C\rangle$ is an extension of the Lebesgue Integral. The uniqueness is the only property that is not immediately apparent. But if $G \in\langle F, A C\rangle$ and $G^{\prime}$ ap $=0$ a.e., then let $Z \subset I$ be the set of points where $G^{\prime}$ ap $\neq 0$. Then $|Z|=0$ and hence $|G(Z)|=0$. Since $G^{\prime}$ ap $=0$ at each point of $I \backslash Z$, it follows that $G(I \backslash Z)=0$ (cf. [2, pp. 225 and 239]). Since $G$ is continuous and $|G(I)|=0$, then $G \equiv C$. In fact, if Lusin's condition $(N)$ is replaced by the following weaker condition (M) the same type of argument goes through.

(M) There does not exist a set $Z$ with $|Z|=0$ and $|F(Z)|>0$ on which $F$ is of bounded variation.

Theorem 1. If $F$ is continuous and $F^{\prime}$ ap exists a.e. and if every $G \in\langle F, A C\rangle$ satisfies (M), then $\langle F, A C\rangle$ is an extension of the Lebesgue Integral.

Proof. As above, it only remains to show the uniqueness of the integral. Thus, if $G \in\langle F, \mathrm{AC}\rangle$ and $G^{\prime}$ ap $=0$ a.e. and $G$ is not identically constant, then there exists $\left[x_{0}, x_{1}\right]$ so that $G\left(x_{0}\right) \neq G\left(x_{1}\right)$. For each $y$ between $G\left(x_{0}\right)$ and $G\left(x_{1}\right)$ write $x(y)=$ $\inf \left\{x \mid x \in\left[x_{0}, x_{1}\right]\right.$ and $\left.G(x)=y\right\}$. It is easily seen that $G$ is monotone on the set $E$ of numbers $x(y)$. Let $Z=\left\{x \in E \mid G^{\prime} \operatorname{ap}(x) \neq 0\right.$ or $G^{\prime}$ ap $(x)$ does not exist $\}$. As before, since $G^{\prime}$ ap $=0$ at each point of $E \backslash Z,|F(E \backslash Z)|=0$. Since $|Z|=0$ and $|G(Z)|=\left|G\left(x_{0}\right)-G\left(x_{1}\right)\right|$ it follows that $G$ does not satisfy (M). This contradiction shows that $G$ is identically constant.

It is well known that a function $\boldsymbol{H}$ that is continuous and of bounded variation, but not absolutely continuous, cannot be used to extend the Lebesgue Integral. This follows from the fact that $H(b)-H(a) \neq \int_{a}^{b} H^{\prime} d x$ on an appropriately chosen interval $[a, b]$. This fact will be improved below to the converse of Theorem 1 .

An extension $\mathcal{Q}$ of the Lebesgue Integral will be considered to be ambiguous if there exist $G_{1} \in \mathbb{Q}, G_{2} \notin \mathbb{Q}, G_{1}-G_{2} \not \equiv C, G_{1}^{\prime}$ ap $=G_{2}^{\prime}$ ap a.e. and $\left\langle G_{2}, A C\right\rangle$ also an extension. By applying the maximality principle it follows that every extension of the Lebesgue Integral is contained in a maximal extension. Let $\mathscr{N}$ be the collection of all maximal extensions and let $G_{0}$ be the intersection of all the elements $\Re$. Then $g_{0}$ is an integral and $g_{0}$ is not an ambiguous extension. To see why, suppose $F_{1} \in g_{0}, F_{2} \in g_{0}, F_{1}-F_{2} \not \subset C, F_{1}^{\prime}$ ap $=F_{2}^{\prime}$ ap a.e. If $\left\langle F_{2}, A C\right\rangle$ is an extension, then $F_{1}$ cannot belong to any extension of $\left\langle F_{2}, \mathrm{AC}\right\rangle$. This contradicts the fact that $F_{1}$ belongs to every maximal extension of the Lebesgue Integral. That there are functions in addition to the AC functions in the integral $g_{0}$ is shown by the following theorem.

THEOREM 2. The primitives for the wide sense Denjoy Integral (ACG functions) are contained in $g_{0}$. 
Proof. The proof of this theorem follows from the fact that the method [2, pp. 254-259] used for the constructive definition of the Denjoy Integral can be applied to any integral. That is, if $\mathbb{Q}$ is a maximal integral, then $\mathcal{Q}$ is closed under the following two operations (otherwise, $\mathbb{Q}$ could be extended by applying these operations).

(c) If $F$ is continuous on $I=[a, b]$ and $a=x_{0}<x_{1}<\cdots<x_{n}=b$ and there exist functions $F_{0}, F_{1}, \ldots, F_{n} \in \mathbb{Q}$ on each closed subinterval of $\left[x_{i-1}, x_{i}\right]$, and $F=F_{i}$ on $\left[x_{i-1}, x_{i}\right]$ then $F \in \mathbb{Q}$.

(h) If $P$ is a perfect set, $P \subset[a, b], a, b \in P, I_{k}=\left[a_{k}, b_{k}\right]$ are the intervals contiguous to $P, F$ restricted to each $I_{k}$ is in $Q, \Sigma\left|F\left(I_{k}\right)\right|<\infty$ and $F(x)-F(a)=$ $\int_{a_{k}}^{x} F^{\prime}$ ap $(x) d t+\Sigma^{\prime} \int_{I_{k}} F^{\prime}$ ap $(x) d t$ where $\Sigma^{\prime}$ is over all $I_{k}<x$ and $x \in\left[a_{k}, b_{k}\right]$, then $F \in \mathbb{Q}$.

Since the transfinite application of these methods of extending an integral do extend the Lebesgue Integral to the Denjoy Integral (i.e., extend the AC functions to the ACG functions) it follows that the ACG functions are in every maximal extension and thus in $\mathscr{G}_{0}$.

Now suppose that $F$ does not satisfy $(M)$ but $F$ is continuous and approximately derivable a.e. Then there is a set $Z$ with $|Z|=0,|F(Z)|>0$ and such that $F$ is of bounded variation on $Z$. A perfect set $P$ can be chosen so that $P$ has the same properties as $Z$ (cf. [1]). Let $F_{2}$ be equal to $F$ on $P$ and linear on intervals contiguous to $P$. Then $F_{2}$ is of bounded variation but is not absolutely continuous. As noted before, the Lebesgue Integral cannot be extended using $F_{2}$. Let $F_{3}=F-$ $F_{2}$. Then any extension containing $F$ cannot contain $F_{3}$. If $P$ is contained in $[a, b]$ and $I_{k}$ are the intervals contiguous to $P$ then $F_{3}$ restricted to $I_{k}$ is an integral in any extension containing $F$. Moreover $F_{3}$ is continuous and $F_{3}\left(I_{k}\right)=0$. It follows from the operation (h) that any maximal extension that contains $F$ must contain $F_{3}$. Since no extension can contain $F$ and $F_{3}$, it follows that there is no extension which contains $F$. Thus the converse to Theorem 1 is true; namely

Theorem 3. If $F$ is continuous and $F^{\prime}$ ap exists a.e. and if there exists a $G \in\langle F, A C\rangle$ such that $G$ does not satisfy (M), then $\langle F, A C\rangle$ is not an extension of the Lebesgue Integral.

THEOREM 4. The following properties characterize the integral $\Phi_{0}$ :

(0) $F \in g_{0}$ implies $F$ is continuous and approximately derivable almost everywhere;

(A) if $G \in\langle F, A C\rangle$ then $G$ satisfies (M);

(E) for every $h$ which is continuous of bounded variation but not absolutely continuous there exists $G \in\langle F+h, A C\rangle$ such that $G$ does not satisfy (M);

(I) if $g$ satisfies (A) and (E), then so does $F+g$.

Proof. The continuity and approximate derivability are by definition.

(1) $F \in g_{0} \Rightarrow F$ satisfies (A). This follows from Theorem 3; for if $F$ does not satisfy $(\mathrm{A}),\langle F, \mathrm{AC}\rangle$ is not even an extension.

(2) $F \in g_{0} \Rightarrow F$ satisfies (E). If not, there exists an $h$ such that for every $G \in\langle F+h, \mathrm{AC}\rangle G$ satisfies (M). From Theorem 1 it follows that $\langle F+h, \mathrm{AC}\rangle$ is an extension. But a maximal extension of $\langle F+h, \mathrm{AC}\rangle$ cannot contain $F$ or it 
would have to contain $h$. This contradiction implies that $F$ satisfies (E).

(3) $F \in g_{0} \Rightarrow F$ satisfies (I). Suppose $g$ satisfies both (A) and (E). Then $\langle g, A C\rangle$ is an extension. Since $F$ is in every maximal extension, $\langle F+g, A C\rangle$ is also an extension and $F+g$ satisfies (A). Suppose $F+g$ does not satisfy (E). Then there exists a continuous $h$ of bounded variation such that for every $G \in\langle F+g+$ $h, \mathrm{AC}\rangle, G$ satisfies $(\mathrm{M})$. Then $\langle F+g+h, \mathrm{AC}\rangle$ is an extension of the Lebesgue Integral. Since $F$ belongs to every maximal extension $\langle g+h, \mathrm{AC}\rangle$ is also an extension. Since $g$ satisfies (E), there exists $G_{0}$ in $\langle g+h, A C\rangle$ such that $G_{0}$ does not satisfy (M). This contradicts the fact that $\langle g+h, \mathrm{AC}\rangle$ is an extension.

(4) To show that all continuous functions that satisfy (A), (E), and (I) and are approximately derivable a.e. are in $G_{0}$, it suffices to show that these properties describe an extension of the integral. From the proof of Theorem 1 it follows that functions that satisfy (A) enjoy the required uniqueness of $F^{\prime}$ ap up to the additive constants. If $F$ satisfies (A), (E), and (I) so does $c \cdot F$. If $F_{1}$ and $F_{2}$ satisfy these three properties, then if $g$ satisfies both (A) and (E) it follows that $F_{2}+g$ satisfies both (A) and (E) and thus $F_{1}+F_{2}+g$ satisfies both (A) and (E) and hence $F_{1}+F_{2}$ satisfies (I). Since (I) implies that $F_{1}+F_{2}$ satisfies both (A) and (E), it follows that functions enjoying these properties form a vector space. If $L$ is a linear change of variables and $F$ satisfies (A), (E), and (I) so does $F(L)$. Finally restriction to subintervals $[a, b]$ of a function which satisfies (A), (E) and (I) does so also. Both (A) and (I) are clearly satisfied; (E) holds true because constant functions satisfy (E) and either $G$ exists interior to subintervals $[a, b]$ or it can be taken to be $h$ if $h$ is of bounded variation but not absolutely continuous on either $(-\infty, a]$ or on $[b, \infty)$.

Theorem 4 gives a characterization of $g_{0}$ which does not involve the maximality principle. However, whether $G_{0}$ properly contains the ACG functions is not known to the author.

\section{REFERENCES}

1. J. Foran, A note on Lusin's condition (N), Fund. Math. 90 (1976), 181-186.

2. S. Saks, Theory of the integral, 2nd rev. ed., Dover, New York, 1937.

Department of Mathematics, University of Missouri-Kansas City, Kansas Ctry, Missouri 64113 